\title{
Design of Tool Automatic Identification and Database Management System Based on RFID
}

\author{
Xiulin Sui ${ }^{*}$, Yan Teng, YongQiu Chen and XueHui Wang \\ School of Mechanical and Power Engineering Harbin University of Science and \\ Technology Harbin, China \\ *suixiulin@sina.com,tengyan996309937@126.com, chenyongqiu@163.com, \\ wangxuehui@126.com
}

\begin{abstract}
RFID (Radio Frequency Identification) is a non-contact automatic identification technology, without human intervention. It is convenient to apply and it can identify fast moving objects. Around the tool and the tool information, the problem of low operational efficiency and low degree of tool management information are still unsolved. In this paper, RFID technology is applied in the tool automatic identification and management system. The tool automatic identification and database management system based on RFID is built. The application model, tool information database design and Upper Computer management software of the RFID in tool identification and management are analyzed. The overall architecture and the software function module of the system are constructed. The database management system is developed by the LabVIEW software. The interface design between LabVIEW and Access database is completed by using ActiveX, as a result, the information of Access database can be written-in and read-out by the management system. Meanwhile, the communication protocol between the Upper Computer and Lower Computer is programed that based on VISA. The RS232 is applied to make the communication between the Upper Computer and Lower Computer come true. Finally, take the SECO's Solid Carbide Ball-end Milling Cutter as an example to have an experiment. Through the experiment, the system proved to be with high reliability and efficiency.
\end{abstract}

Keywords: RFID, Tool Identification, Database Management, LabVIEW

\section{Introduction}

The tool as the most important resource consumption of modern manufacturing industry attracts much attention. Generally speaking, the tool costs account for 3\% to 5\% of the manufacturing cost. However, relevant data show that $45 \%$ to $55 \%$ of the tool is unused for a long time due to the lack of effective tool management. Therefore, it is urgent for modern manufacturing industry to build a reliable and efficient way of tool identification and database management system. Besides, it's important to reduce the production cost and improve the production efficiency. Recently, domestic and foreign researchers have studied the tool procurement, access management, tool preparation and tool information. Tool management method is proposed and the corresponding software system is developed. But we should pay more attention to the identification and database management systems of tool. In this paper order to achieve the rapid recognition and effective management of tool information database, the RFID technology is applied in the Quick identification of tool. Through Access the tool information database is built, the tool length, blade length/blade length, speed and other information are stored into the database with a fixed format; the database management system 
is programed by LabVIEW and the Access database is called through ActiveX controls, as a result, manage the variety of tool information comprehensively can be achieved. The relevant information can be always queried via the tool management system by users, the efficiency of tool preparation and use can be improved.

\section{Design of System Structure}

\subsection{Composition of System Structure}

The structure of this system mainly includes: RFID system, Upper Computer management software, Access tool information database. Among them, RFID system consists of RFID tag, reader and other components; Programing the Upper Computer management software and managing tool database by LabVIEW.

\subsection{Working Principle of System}

The system is designed to achieve the following three functions: 1) Tool information management function. LabVIEW Upper Computer software is applied to realize the read and write operation of Access tool information database through the ActiveX controls, and to complete the query and display operation of tool information; 2) Tool identification function of tool library. That mainly is to realize the query and recognize of the tool information, and realize the quick selection of random storage tool in the tool library. 3) Quick input and read of the tool information. The tool code which is generated from the Upper Computer can be written into the electronic tag according to a certain format by RFID reader. Meanwhile, the reader can quick read the tool information through electronic tag.

\section{Design of RFID System}

\subsection{Composition of RFID System Structure}

Composition of RFID system structure is shown in Figure 1.

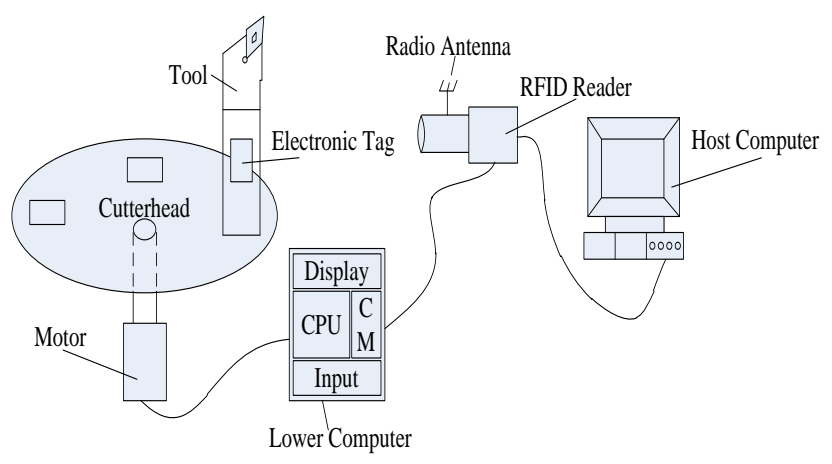

Figure 1. Composition of RFID System Structure

RFID(Radio Frequency Identification) is a non-contact automatic identification technology, which can obtain relevant information through the RF signal recognize the target automatically and do not need contraction and optical visual display[1]. The RFID system mainly includes Electronic Tag and Reader. The basic working principle of RFID system is shown in Figure 2. 


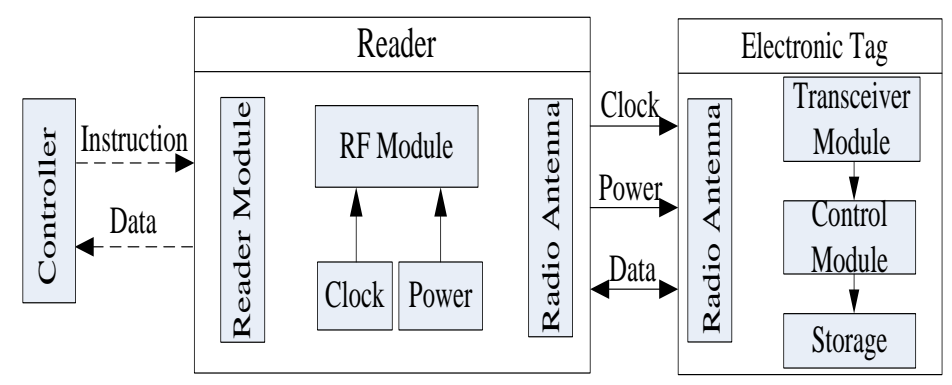

Figure 2. Working Principle of RFID System

Electronic Tag, also known as smart label, it is a super mini small label. Which is composed of IC chip and wireless communication coil, its built-in radio antenna is used for communicating with the reader. Electronic Tag is the carrier of data. The tool length, material, manufacturer and other information of the tool are written into the electronic tag with a fixed format. The uniqueness can easy to be achieved. An electronic tag represents a unique tool. The Philips M1 passive anti-metal tag is selected as the tool tag, the tag performance is shown in Table 1. The electronic tag is placed on the hilt, which is easy to read and detect for the reader.

Table 1. Tag Performance

\begin{tabular}{c|c}
\hline Tag parameters & Product Performance Description \\
\hline Chip & Philips Mifarel \\
\hline Working Frequency & $13.56 \mathrm{MHz}$ \\
\hline Boundary Dimension & $16 \mathrm{~mm}^{*} 1 \mathrm{~mm}$ \\
\hline Protocol Standard & ISO14443A/B \\
\hline Power Supply Mode & Passive Electronic Tag \\
\hline Read-Write Equipment & PCD \\
\hline The maximum reading distance & $100 \mathrm{~mm}$ \\
\hline
\end{tabular}

Reader is also called reader, it is a bridge to contact the computer and electronic tag, it usually consists of RFID module, read/write module, antenna. It is responsible for reading data of electronic tag, and passing the data to the Upper Computer. We choose the BISC-600007-650-00-k11 reader of BALUFF Company. The reader is placed on a moving probe, that we can read the electronic tag data by controlling the movement of the probe.

\subsection{Working Principle of RFID System}

The work method is controlled by the read-write command which come from Upper Computer through the database management system sent to the reader. The electronic tag stores the object information which to be identified. When in the radio working range of reader antenna, the electromagnetic wave (command, energy, and timing) is sent from reader to electronic tag, induced current is generated from electronic tag antenna. And thus the electronic tag is activated after gain the energy. The electronic tag execute the commands and itself encoded information is sent out by the built-in radio antenna; The reader antenna receives a modulated signal which is sent from the tag and then the tag information is transmitted to the reader processing module. After demodulation and decoding the effective information is sent to the Upper Computer software system to process. The reader realizes the read and write of tag identification code and memory data, and read out the data is sent to the Upper Computer through the serial port for analysis and processing. 


\subsection{Communication between RFID and Upper Computer}

Classic and traditional RS232 communication mode is adopted to communicate between RFID and Upper Computer; the Access database is called through ActiveX controls to control the data. The RS232 is the general communication mode between Upper Computer and Lower Computer. Serial communication is widely used in the field of communication. Standard RS232 interface has become a standard interface of the computer, peripherals, switches and many communication devices. Meanwhile standard RS232 is widely used in micro-computer system and large system, it also has the advantages of simple attachment, long communication distance and so on. The Lower Computer sent the tool information which is collected by RFID module to the Upper Computer through RS232. After treated by the Upper Computer, the data is written in Access or the tool information is retrieved from the Access database and then displays it. The flow diagram of the RS232 serial communication is shown in Figure 3.

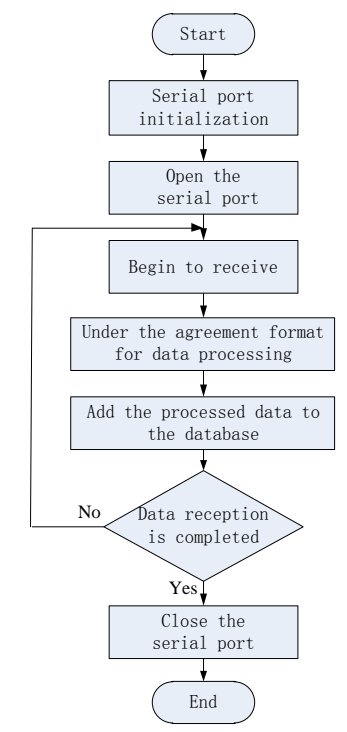

Figure 3. Flow Diagram of the RS232 Serial Communication

\section{Design of Database}

The demand of the user is reflected in input, save, modify and query of all kinds of information. We require the database structure can satisfy input and output all kinds of information. Data, data structures and the flow of data processing are the basis design of the database. Analyzing the business process of tool management system, designing the data item and data structure are shown in the following [2].

Tool information library: tool name, tool number, tool material, tool diameter, tool length, manufacturers and so on.

Tool matching information library: Tool number, tool name, tool parameters, tool attribute, matching components.

Tool inventory information library: Tool procurement, tool stock-out, tool repair, tool inspection, tool scrap, inventory alarm, tool selection list and so on. 


\subsection{Design of Database Concepts}

After getting the data item and data structure, various entities can be designed to meet the users' requirements, and the relationship between them to build the base for the design of logical structure. These entities include several of specific information. The stream of data is formed by the action between each other. Tool information input entity diagram is shown in Figure 4.

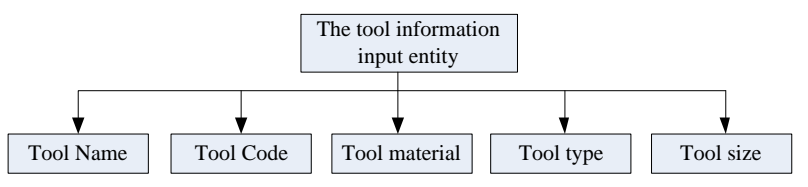

Figure 4. Input Entity Diagram for Cutting Tool Information

\subsection{Design of Database Logical Structure}

The design of database structure is transmitted into the data model which is supported by Access database system. It is also a logical structure of the database [3]. The tool information input table is shown in Figure 5, components supporting table is shown in Figure 6, Tool properties table is shown in Figure 7 and Access table is shown in Figure 8.

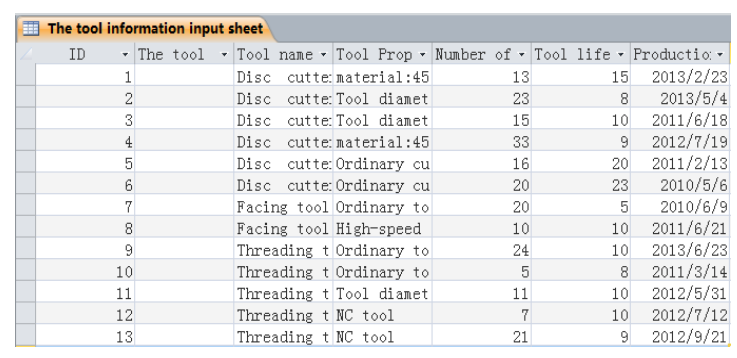

Figure 5. Tool Information Input Table

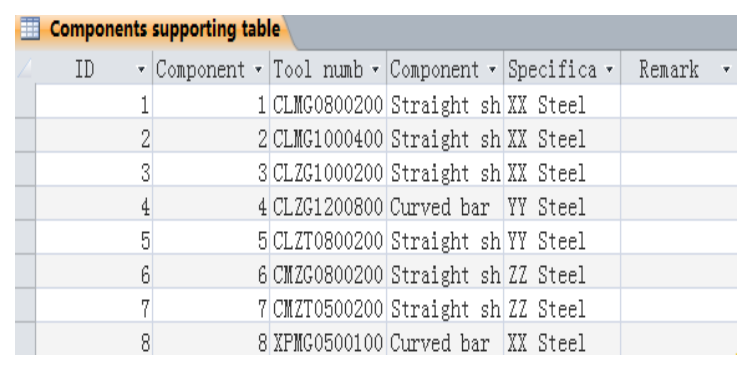

Figure 6. Components Supporting Table

\begin{tabular}{|c|c|c|c|c|}
\hline \multicolumn{5}{|c|}{ Tool properties table } \\
\hline ID & - Tool attr. & Tool numb - & Tool name & - Tool Prop. \\
\hline 1 & 1 & CLZG0800100 & Threading tool & IC lathe \\
\hline 2 & 2 & XMZZ1000200 & Facing mill & NC milling \\
\hline 3 & 3 & XPIG0800200 & Disc mill & $\mathbb{I C}$ milling \\
\hline 4 & 4 & XIMT0600100 & Facing mill & $\mathbb{N C}$ milling \\
\hline 5 & 5 & CLZG1000400 & Threading tool & $\mathbb{N C}$ lathe \\
\hline 6 & 6 & CYZG0800200 & indrical shank To & Io NC lathe \\
\hline
\end{tabular}

Figure 7. Tool Properties Table 


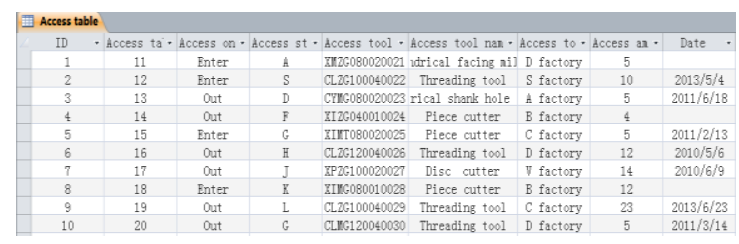

Figure 8. Access Table

From above four tables, tool information input table can make connection with "out / in number" of Access table by "tool code", components supporting table and "tool code" of Tool properties table. Then the tool can be tracked all the time by using tool code. As a result, the internal relation diagram of tool database can be obtained, as is shown in Figure 9.

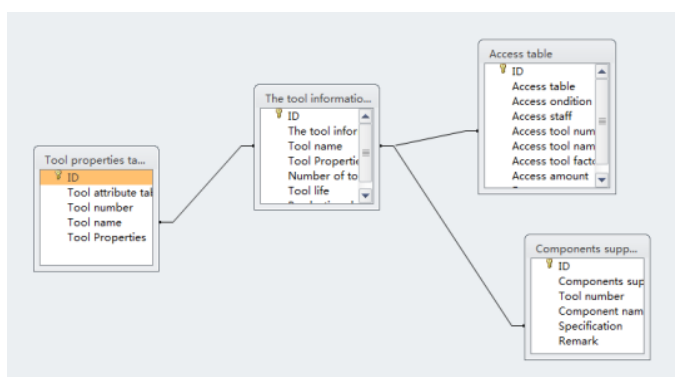

Figure 9. Relationship of the Internal Database

\section{Design of Tool Management System}

Based on the short development cycle, practical, and many other advantages of LabVIEW, the Upper Computer communication software is programed by LabVIEW 2011 software. The interface design between LabVIEW and Access, and the writing of the serial communication protocol between LabVIEW and Lower Computer are all programed by LabVIEW2011 software. The main function is the corresponding tool information can be gotten through the Upper Computer interface. Meanwhile the command which needs to be executed is sent by the serial communication, then reading the tool electronic tag and processing the read information. Besides, the Access database information is called by the management interface. Or transmitting the tool information to the reader by serial port, and then writing in electronic tag by the reader.

\subsection{Division of System Function Modules}

The whole using process of tool will be tracked and managed by the tool database management system which is developed in this paper. Including tool code, tool query, tool stock-in/out and so on are all tracked and managed. Therefore, the system automatically generates a unique tool code and record the basic information, parameter information and so on when the new tools stored in the library. While the tool administrator can also edit the relevant information that needed, and the system can also have different queries according to different needs. To meet the needs of the tool management, the system needs to make a count to the tools in library in different ways according to specific needs [4]. And it needs to have the function of generating reports. While the tool operator can record the tool lend and return information, and tracking and recording the inventory, tool life and other information. In order to maintain the system, the management system need to have the functions of adding users, change users, using log management, system maintenance and so on. 
According to the above design ideas of the overall system, the system is designed into two sub-modules: Tool resource management and basic data management, as shown in Figure 10.

Tool resource management includes:

1) Tool management: To achieve the management operations of tool stock-in, stock-out, scrap, lending, return and so on;

2) Tool query: To achieve the tool quick query, includes two ways of manually enter the tool code and handheld RFID reader scan the tool tag.

Basic data management function includes:

1) System management: To achieve the user management, authority management, system data replica and other functions.

2) Tool code: To achieve the setting, input, maintenance, query and other functions of the tool coding principles;

3) System help: To describe the system function, usage, precautions, and the tool coding rules of the system in detail, it is easy to use for the users [5].

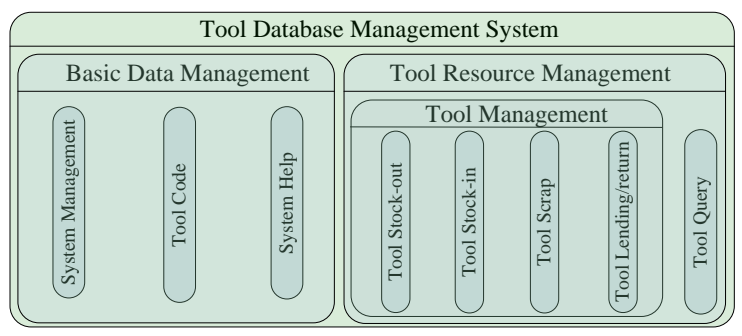

Figure 10. System Function Module

\subsection{Interface Design between LabVIEW and Access}

Use the ActiveX of LabVIEW to visit the tool information database which is constructed by Access and the database includes four fields: Tool code, Tool Type, Tool Manufacturer and Tool Material. The effect of the completed program is that the users select code on the front panel, while pressing the Query to obtain other corresponding tool information, thus the basis of the tool selection can be provided. Here only take tool information query as an example to introduce the interface between LabVIEW and Access, the design of LabVIEW program block diagram is shown in Figure 11.

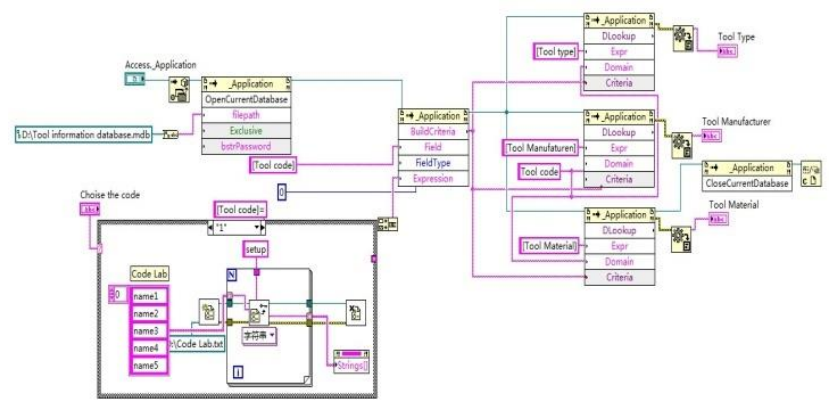

Figure 11. Design of LabVIEW Program Block Diagram

When using the Automation Open, Automation Refnum (automatic link parameters) is the first should be assigned. ActiveX/Automation Refnum is selected in the control module, after adding clicks the mouse button and chooses Select ActiveX Class/ Browse. At this moment a 
dialog box is open, selects "Microsoft Access 14.0 Object Library Version 14.0, then selects Application in the Objects. Finally, the ligature work can be completed in the block diagram program, thus the designation of link type can be realized.

There are many methods to query the database. We can query by parameter, the corresponding record is retrieved according to the query rule which is specified by the users. After querying by using the Dlookup method of Open Current Database, the corresponding records can be output. When users press Query key, the program can obtain the corresponding data of Tool Type, Tool Manufacturer, Tool Material according to query Tool Code of the database, thus it need to be connected respectively[6]. After finishing all above query and display, the current database need to be closed.

\subsection{RS232 Serial Communication}

VISA (Virtual Instrument Software Architecture) is a high-level application programming interface which is developed by the U.S. National Instrument company for communicating with all kinds of Instrument bus. The VISA library resides in the computer system to complete the connection of software between the computer and the instrument, so the control of the instrument procedures can be realized.

The serial communication sub-VI of VISA of LabVIEW is used to realize the communication between the Upper Computer and the Lower Computer. First, the VISA Configure Serial Port.vi is used to initialize the serial port, setting the baud rate to 9600,8 data bits, and white parity bit, 1 stop bit with no flow control [7]. Then open the serial port, and the data of the electronic tag is transmitted from the RFID reader to the Upper Computer by the serial port. As a result, the Upper Computer query and display the tool's information according to the pre-programed procedure. The tool code which generated from the Upper Computer is transmitted to the RFID reader by serial port, and the tool code is written in the electronic tag meanwhile the reader is controlled, VISA serial communication protocol block diagram is shown in Figure 12.

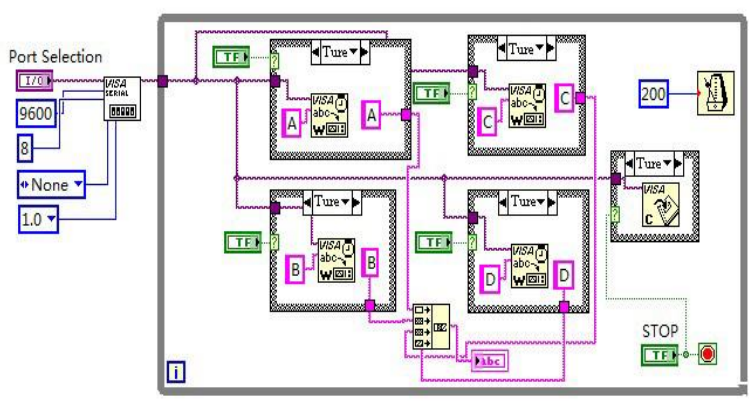

Figure 12. VISA Serial Communication Protocol Block Diagram

\section{Functional Verification of System}

The work flow of tool information query and tool stock-in/stock-out is shown in Figure 13. 


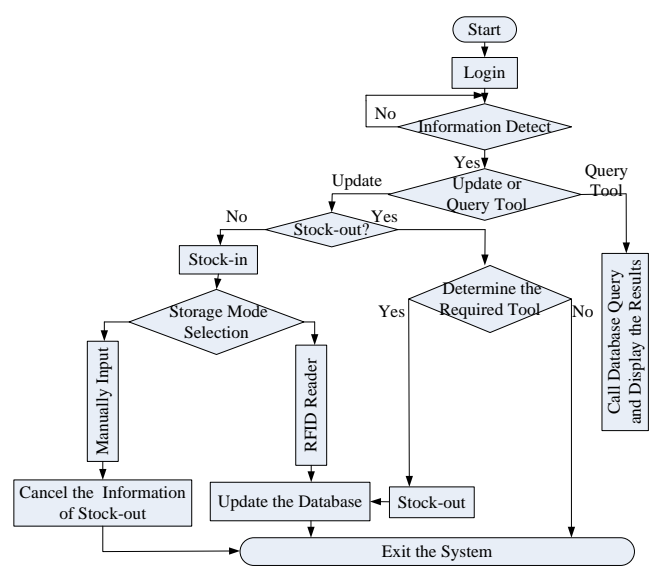

Figure 13. Work Flow of Tool Information Query and Tool Stock-in/Stock-out

1) After starting the tool management system, by entering the user information, checking this user data information whether matching with the user information data table of background information database. If the check is successful this data information will be stored into the specified database user login information table [8];

2) After the system confirms the identity of the user, the user can access the corresponding function. If select the tool query can follow the specified tool coding to query specific information of a tool;

3) The tool updates include tool stock-in and stock-out. When lending the needed tool from the library, the production line code, tool inventory code, tool lending time, the number of lending and other information will be written in the tool lending table by system. Tool stockin includes new tool stock-in and returned tool stock-in. When the operation of new tool is stock-in, the basic information tables of database and other related records will be updated. The same as the tool code, tool classification, tool material, tool manufacturer, tool size and so on.

In order to verify the accuracy and stability of the system, the Solid Carbide Ball-end Milling Cutter (970051-TRIBON) that produced by SECO is selected to validate the system performance [9]. The tool parameters are shown in Table 2. A new tool coding, identification, stock-in, query and other functions are shown in this experiment. Firstly, fix the Philips M1 passive anti-metal tag on the hilt. Then input the related information in the tool coding subsystem, which is shown in Figure 14. When the users click the Generating Code button, the system can generate the tool code with a certain format. Meanwhile, the tool code is sent to the RFID reader through the serial. Finally, users can write the tool coded into the electronic tag by the reader. 


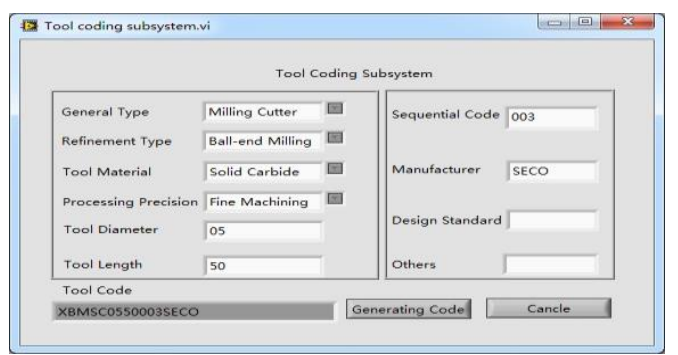

Figure 14. Tool Coding Interface

Table 2. Tool Parameters

\begin{tabular}{c|c}
\hline Tool Name & Solid Carbide Ball-end Milling Cutter \\
\hline Tool Model & $970051-$ TRIBON \\
\hline Manufacturer & SECO \\
\hline Tool Material & Solid Carbide \\
\hline General Type & Milling Cutter \\
\hline Refinement Type & Ball-end Milling Cutter \\
\hline Processing Precision & Fine Machining \\
\hline Tool Diameter & $5.0 \mathrm{~mm}$ \\
\hline Tool Length & $50 \mathrm{~mm}$ \\
\hline
\end{tabular}

Users can query the relevant information by tool query subsystem (as shown in Fig.15). Access database can be called by the query interface. Manually open the tool query subsystem to select Tool Code and click the Query, and then the corresponding tool information can be obtained; besides, users can also read the tag corresponding tool by handheld RFID reader [10]. Tool code in the electronic tag is transmitted from the RS232 serial port of Lower Computer to the query subsystem of Upper Computer. As a result, the relevant information of tool can be queried more quickly.

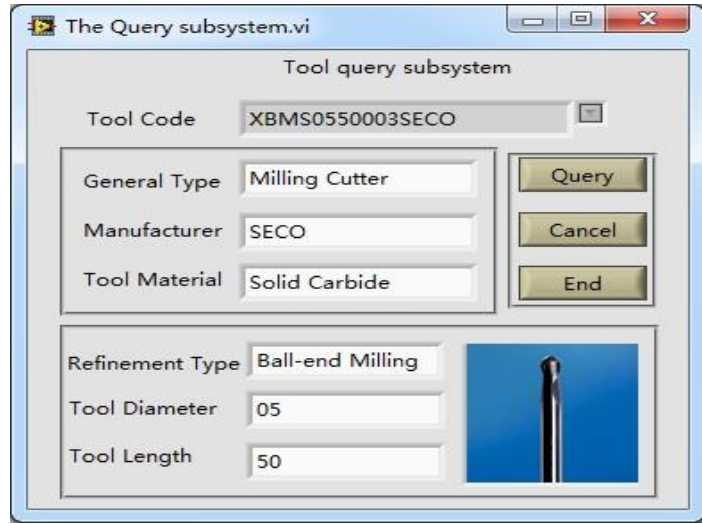

Figure 15. Tool Query Interface

Users can have the operation of tool stock-in and stock-out through the tool management subsystem. All operations are around the tool code in this process. Only select Tool Stockin/out in the management interface. Select the Tool Coding in the pop-up window, and then the operation is completed. It is important to say that the tool stock-in/out operations can be completed through the handheld RFID reader. The tool stock-in/out can be achieved quickly and easily by the reader read the tool code on tool electronic tag. 


\section{Conclusions}

The mature RFID technology is applied in the tool identification management, the function of rapid identification; query and so on is achieved; the tool automatic identification and database management system based on RFID is built Make the system to have requirements analysis, functional design, software process design, software development framework analysis, database conceptual model and the relational model design. LabVIEW and Access are used as the tool to develop the system and database. LabVIEW is also used to develop the system software. This paper focusing on the development of tool coding module, query module, tool management module. Around the tool code, the tool information database is created by using Access, the relevant information of the tool is integrated together, and the tool can be managed conveniently; LabVIEW is used to program the Upper Computer management interface of control system. The design of interface between LabVIEW and the Access database is achieved by ActiveX controls. RS232 as a mature communication technology is widely used in the computer communicate and the master-slave control. RS232 communication between Upper Computer and Lower Computer can be achieved by applying the VISA controls in LabVIEW. The RFID tool identification system controlled by the Upper Computer management software is achieved. And the data can be exchanged by the Upper Computer management software. Finally, take the SECO's Solid Carbide Ball-end Milling Cutter as an example to have an experiment to verify the functions of tool coding, stock-in/ out, tool query. Experiment shows that tool can be recognized quickly and stably by RFID technology and the system works stably. The fact is that the rapid display and input of tool information is realized.

\section{Acknowledgements}

This research was sponsored by Excellent Academic Leaders Project of Harbin science and technology innovation talents of special fund (2013RFXXJ064).

\section{References}

[1] X. G. Gao, Z. Xiang, H. Wang, "An approach to security and privacy of RFID system for supply chain", Proc. IEEE International Con.f E-Commerce Technology for Dy-namic E-Business, (2004).

[2] N. Kalla, "Reliability Test of an RFID System for Tool Management on Construction Sites", US. Texas A\&M University. (2007).

[3] G. X. Wang and Y. Yan, "A methodology of tool lifecycle management and control based on RFID", Proc.IEEE IEEM, (2009).

[4] F. Klaus, RFID Handbook: Fundamentals and Applications in Contactless Smart Cards. Radio Frequency Identification and Near-Field Communication. Wiley. (2010).

[5] J. Travis and J. Kring, "LabVIEW for Everyone: Graphical Programming Made Easy and Fun", Prentice Hall, Crawfor. Crawfordsville\&U.S, (2010).

[6] National Instrument, "LabVIEW User Manual, 2001G. H. Gao, Y. Z. Zhang, Using Activex Technology to Access the Database in LabVIEW", Foreign Electronic Measurement Technology, (2004).

[7] Y. Jiang and W. M. Zhang. "Research on machining center tool information management system based on RFID", Manufacturing Technological \& Machine Tool, (2013).

[8] X. J. Lin, C. Gao, Y. X. Xu, "Database Technology in Tool Management System", Aeronautical Manufacturing Technology, (2013).

[9] D. S. Wang, "Tool Management Function of Siemens Sinumerik 828D", Manufacturing Technological \& Machine Tool, (2012).

[10] F. Pan, "Application of RS 232 Serial Port in Communication between PC and MCU”, Modern Electronics Technique, (2010). 
International Journal of Database Theory and Application Vol.7, No.5 (2014) 